\title{
The effect of intermetallic inclusions on the formation of subsurface cracks in the AlMg6 alloy under very high cycle fatigue
}

\author{
D. A. Bilalov ${ }^{\dagger}$, V. A. Oborin, O. B. Naimark \\ †ledon@icmm.ru \\ Institute of Continuous Media Mechanics UB RAS, 1 Academician Korolyov St., Perm, 614013, Russia
}

\begin{abstract}
The article discusses the process of deformation and fracture of the AlMg6 alloy (a material for aircraft engine building) under very high cycle fatigue. The choice of research material is substantiated, a description of its chemical composition is given. The geometry of samples for fatigue testing, loading conditions and characteristics of testing machines are given. For fatigue tests, a Shimadzu USF-2000 ultrasonic machine was used. Preliminary dynamic loading was carried out on a split HopkinsonKolsky bar. A series of experiments on the fatigue failure of the AlMg6 alloy was carried out, including after preliminary dynamic loading. Analysis of the fracture surfaces revealed two characteristic types of subsurface cracks. Chemical analysis showed that in the first case, the intermetallic inclusion is the focus of destruction, since a change in the percentage ratio of aluminum and magnesium in the test material was observed. In the second case, such a change was not observed, therefore, a conclusion is drawn according to which the internal defect served as the focus of destruction. A mathematical model is proposed for describing the deformation behavior of metals and alloys, based on the statistical theory of defects. Particular attention is paid to the selection of the destruction criterion. The conditions are imposed that the fracture criterion under cyclic loading must satisfy. A criterion is constructed based on the critical value of the accumulated energy. The ideology of determining unknown model parameters is proposed. Identification of unknown parameters was carried out. A number of computational experiments were carried out. The calculations are in good agreement with the experimental data. It is shown that the proposed mathematical model is capable of predicting the fatigue failure of the material under study, taking into account preliminary dynamic effects based on $10^{6}-10^{9}$ cycles.
\end{abstract}

Keywords: very high cycle fatigue, intermetallic inclusions, crack, fracture, endurance limit.

УДК: $539.388 .1 ; 539.431 ; 539.422 .24$

\section{Влияние интерметаллидных включений на образование подповерхностных трещин в сплаве АМг6} при гигацикловой усталости

\author{
Билалов Д. А. ${ }^{\dagger}$, Оборин В. А., Наймарк О. Б. \\ Институт механики сплошных сред УрО РАН, ул. Академика Королёва 1, Пермь, 614013, Россия
}

В статье рассмотрен процесс деформирования и разрушения сплава АМг6 - материала авиамоторостроения при гигацикловой усталости. Обосновывается выбор материала исследования, приводится описание его химического состава. Приводится геометрия образцов для проведения усталостных испытаний, условия нагружения и характеристики испытательных машин. Для усталостных испытаний использовали ультразвуковую машину Shimadzu USF-2000. Предварительное динамическое нагружение проводили на разрезном стержене ГопкинсонаКольского. Проведена серия экспериментов по усталостному разрушению сплава АМг6, в том числе - после предварительного динамического нагружения. При анализе поверхностей разрушения выявлено два характерных типа подповерхностных трещин. Химический анализ показал, что в первом случае очагом разрушения служит интерметаллидное включение, так как наблюдали изменение процентного соотношения алюминия и магния в исследуемом материале. Во втором случае такого изменения не наблюдали, поэтому делается вывод, согласно 
которому очагом разрушения служил внутренний дефект. Предложена математическая модель для описания деформационного поведения металлов и сплавов, базирующаяся на статистической теории дефектов. Особое внимание уделяется выбору критерия разрушения. Накладываются условия, которым должен удовлетворять критерий разрушения при циклическом нагружении. Построен критерий, базирующийся на критическом значении накопленной энергии. Предложена идеология определения неизвестных параметров модели. Проведена процедура идентификации неизвестных параметров. Проведён ряд вычислительных экспериментов. Проведённые расчёты хорошо согласуются с экспериментальными данными. Показано, что предложенная математическая модель способна предсказывать усталостное разрушение исследуемого материала с учётом предварительного динамического воздействия на базе $10^{6}-10^{9}$ циклов.

Ключевые слова: гигацикловая усталость, интерметаллидные включения, трещина, разрушение, предел выносливости.

\section{1. Введение}

Конструкции и механизмы при эксплуатации в ряде случаев претерпевают повторяющиеся нагрузки ниже предела прочности, что в конечном итоге приводит к усталостному разрушению. Выделяют мало-, многои гигацикловую усталость (ГЦУ). В режиме ГЦУ амплитуды приложенных напряжений меньше предела пропорциональности. Таким образом, материал способен выдержат $10^{8}-10^{9}$ циклов. Ключевая особенность разрушения при гигацикловой усталости - это зарождение трещины под поверхностью. При этом большая часть циклов приходится на её образование, a не на рост [1]. Эти две особенности делают указанный вид разрушения опасным из-за сложности диагностики. Поэтому возникает необходимость в изучении механизмов зарождения подповерхностных трещин и в предсказании усталостной долговечности материалов и конструкций на базе $10^{9}$ циклов.

\section{2. Материал и условия эксперимента}

В качестве исследуемого материала был выбран сплав АМг6, используемый в авиастроительной отрасли для внутренних наборов самолётов и топливных баков [2]. Материал имеет высокую устойчивость к коррозии, конструкционную прочность и выносливость [3]. Химический состав АМг6: $\mathrm{Fe}<0.4, \mathrm{Si}<0.4$, $\mathrm{Mn}$ 0.5-0.8, Ti 0.02-0.1, Al 91.1-93.68, $\mathrm{Cu}<0.1$, Be $0.0002-0.005, \mathrm{Mg} 5.8-6.8, \mathrm{Zn}<0.2$.

Испытания на усталость проводили на ультразвуковой машине Shimadzu USF-2000 с частотой нагружения 20 кГц и коэффициентом асимметрии цикла $R=-1$. Геометрия образцов изображена на Рис. 1 . Предварительное динамическое нагружение (растяжение) проводили на разрезном стержне ГопкинсонаКольского [4] с характерной скоростью деформации $10^{3} \mathrm{c}^{-1}$ и величиной деформации от 7 до $15 \%$. Эксперименты по предварительному растяжению проводили на готовых образцах с геометрией, изображённой на Рис. 1. Показано, что в исходном состоянии предел выносливости на базе $10^{9}$ циклов для сплава АМг6 равен 154 МПа. Предварительная динамическая деформация, величиной 7\% снижает его до $136 \mathrm{MПа} \mathrm{(на} \mathrm{12 \% );}$ при деформировании на $10 \%$ предел выносливости снижается до 121 МПа (на 21\%); при деформировании на 15\% - до 112 МПа (на 27\%).

\section{3. Анализ поверхностей разрушения}

В процессе усталостных испытаний часть образцов разрушались образованием трещины с поверхности, часть - с образованием очага разрушения в объёме материала. Среди трещин, образовавшихся под поверхностью, можно выделить два характерных типа (Рис. 2). Первый тип - «рыбий глаз» («fish еуе») более выраженный (Рис. 2 a) и расположен дальше от границы, чем у второго типа (Рис. 2b). На Рис. 2 а цифрой 1 обозначен очаг разрушения, 2 - область внутри «рыбьего глаза», 3 - область нестабильного роста трещины. Размер «рыбьего глаза» (диаметр второй области) на Рис. 2 a -2.4 мм, а на Рис. 2 b -0.5 мм.

Химический состав исследуемого материала определялся с помощью сканирующего электронного микроскопа Hitachi S-3400n по спектру излучения отраженных электронов с помощью модуля INCA.

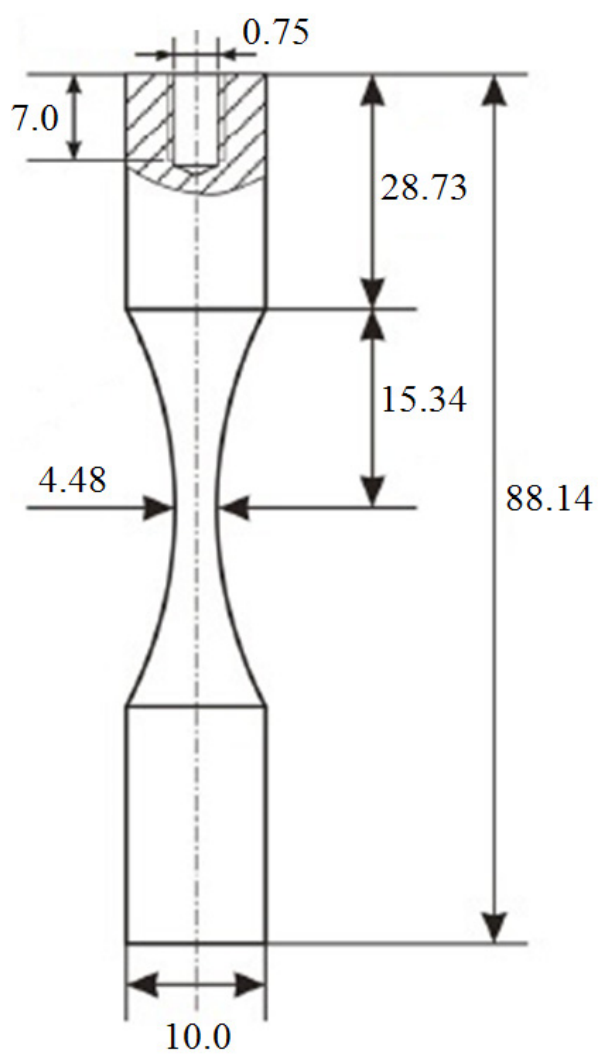

Рис. 1. Геометрия образцов для испытаний на усталость.

Fig. 1. The geometry of the samples for fatigue tests. 


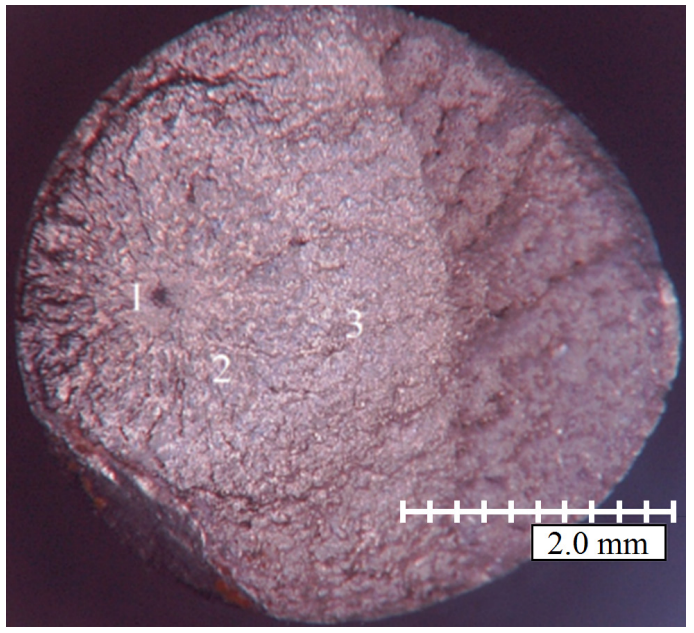

a

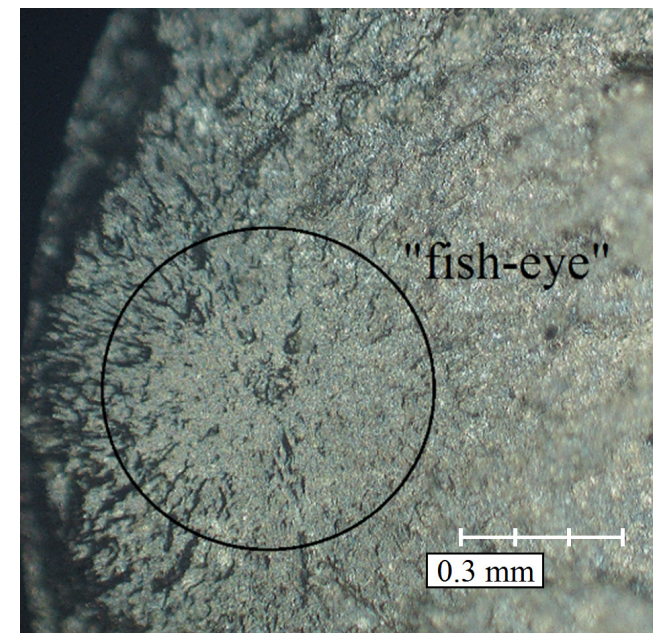

$\mathrm{b}$

Pис. 2. (Color online) Характерные типы подповерхностных трещин. Амплитуда нагружения 120 МПа, образец разрушился через

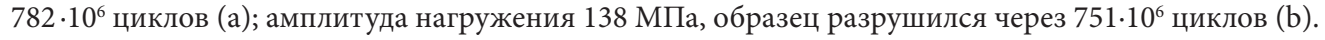

Fig. 2. (Color online) Typical types of subsurface cracks. The amplitude of loading is $120 \mathrm{MPa}$, the specimen broke after $782 \cdot 10^{6} \mathrm{cycles}(\mathrm{a})$; the amplitude of loading is $138 \mathrm{MPa}$, the specimen broke after $751 \cdot 10^{6}$ cycles (b).

Химический анализ поверхности разрушения показал, что для первого типа подповерхностной трещины характерно изменение химического состава вблизи очага разрушения (Рис. 3). В области 2 (Рис. 3) содержание $\mathrm{Al}-49 \%, \mathrm{Mg}-42 \%$, в области 3-72 и $24 \%$ соответственно. В области $1-94 \% \mathrm{Al}$ и $6 \% \mathrm{Mg}$, как и положено для сплава АМг6. Для второго типа «рыбьего глаза» существенного изменения химического состава в очаге разрушения не наблюдали.

Указанное изменение химического состава даёт основание полагать, что концентратором накопления повреждённости стало интерметаллидное включение $\mathrm{Al}_{52} \mathrm{Mg}_{48}$ (Ц-фаза), характерное для сплавов системы $\mathrm{Al}-\mathrm{Mg}$ [5]. Г-фаза является наиболее близкой по составу по сравнению с другими возможными: $\mathrm{Al}_{3} \mathrm{Mg}_{2}$ ( $\beta$-фаза), $\mathrm{Al}_{12} \mathrm{Mg}_{17}$ ( $\gamma$-фаза), $\mathrm{Al}_{30} \mathrm{Mg}_{23}$ (є-фаза). Действительно, процентное соотношение элементов в составе 丂-фазы - 54.6\% алюминия и $45.4 \%$ магния, а в очаге

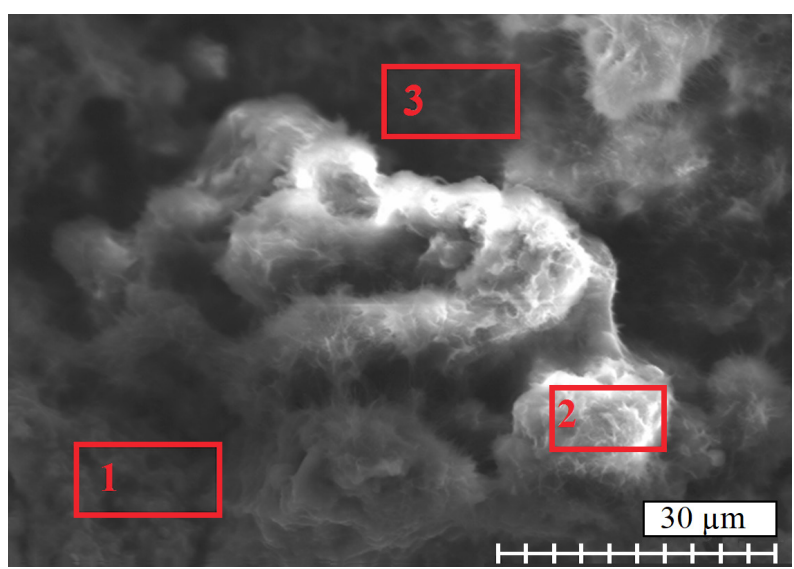

Рис. 3. Характерные области очага разрушения подповерхностной трещины первого типа.

Fig. 3. Characteristic areas of the fracture zone of a subsurface crack of the first type. разрушения 53.8\% (49/0.91) и 46.2\% (42/0.91) соответственно. Что касается области 3 на Рис. 3, то она по своему составу близка к эвтектике $\mathrm{Al}+\mathrm{Al}_{3} \mathrm{Mg}_{2}$ [5].

В работах [6-8] отмечается, что подповерхностные трещины могут зарождаться как на включениях, так и на внутренних дефектах (микропоры, микротрещины, микросдвиги и т.д.). Таким образом, можно сделать вывод, что для второго типа «рыбьего глаза» (Рис. 2b) зародышем очага разрушения стал внутренний дефект.

\section{4. Математическое моделирование}

В рамках работы проведено моделирование процесса разрушения сплава АМг6 при комбинированных динамических и усталостных воздействиях. Предложена модификация определяющих соотношений [9], для описания деформационного поведения металлов и сплавов:

$$
\begin{gathered}
\dot{\varepsilon}^{p}=\dot{\varepsilon}_{0}^{n_{\varepsilon}}\left(\Gamma_{\sigma} \sigma-\Gamma_{p \sigma} \frac{\partial F}{\partial p}\right), \\
\dot{p}=\dot{\varepsilon}_{0}^{n_{p}}\left(\Gamma_{p \sigma} \sigma-\Gamma_{p} \frac{\partial F}{\partial p}\right), \\
\frac{F}{F_{m}}=\frac{p^{2}}{2}-\frac{p^{2}}{2 \delta}+c_{1} p+c_{2} \ln \left(c_{3}+c_{4} p+p^{2}\right)-\frac{\sigma p}{2 G},
\end{gathered}
$$

где $\varepsilon^{p}-$ пластическая деформация; $\dot{\varepsilon}_{0}-$ характерная скорость деформации (интенсивность тензора скорости деформации); $\Gamma_{\sigma}, \Gamma_{p \sigma}, \Gamma_{p}-$ положительные кинетические коэффициенты; $\sigma-$ приложенное напряжение; $F$ - неравновесная свободная энергия; $p$ - параметр плотности микродефектов; $n_{\varepsilon}, n_{p}-$ константы, отвечающие за скоростную чувствительность материала; $G-$ модуль сдвига; $F_{m}, \delta, c_{1}-c_{4}-$ константы аппроксимации. В одномерной одноосной постановке $\varepsilon^{p}, \sigma, p-$ соответствующие компоненты тензоров $\boldsymbol{\varepsilon}^{p}, \boldsymbol{\sigma}, \boldsymbol{p}$. 
Ключевым моментом при моделирования разрушения при комбинированном нагружении является учёт накопления повреждённости. Для используемой модели ранее были предложены критерии разрушения на основе инвариантов [10] и интенсивностей [9] тензора плотности микродефектов, а также на основе накопленной энергии [11]. Однако все они имеют общий недостаток: поврежденность, согласно данным критериям, не является монотонно возрастающей функцией процесса деформирования. При разгрузке она ниспадает. Поэтому такие критерии можно адекватно использовать лишь при активном нагружении. Для адекватного предсказания разрушения при циклических нагрузках, с учётом предварительного динамического нагружения, предложен модифицированный критерий на основе накопленной энергии, учитывающий всю предысторию деформирования.

Согласно [11], источник тепла при неупругом деформировании может быть описан следующим соотношением:

$$
\dot{Q}=\sigma \dot{\varepsilon}^{p}-\frac{\partial F}{\partial p} \dot{p}=\sigma\left(\dot{\varepsilon}^{p}+\dot{p}\right)-\frac{\partial \Psi}{\partial p} \dot{p},
$$

где $\partial F / \partial p=(\partial \Psi / \partial p)-\sigma ; \sigma\left(\dot{\varepsilon}^{p}+\dot{p}\right)-$ работа неупругого деформирования; $(\partial \Psi / \partial p) \dot{p}-$ запасённая энергия. Тогда коэффициент Тейлора-Квини (доля диссипированной энергии при неупругом деформировании) примет вид:

$$
\beta=1-\frac{(\partial \Psi / \partial p) \dot{p}}{\sigma\left(\dot{\varepsilon}^{p}+\dot{p}\right)},
$$

a доля запасённой энергии соответственно равна $1-\beta$. Для учёта предыстории деформирования проинтегрируем выражение (5) по деформациям:

$$
\omega=\int_{0}^{\varepsilon_{c}}(1-\beta) d \varepsilon,
$$

где $\omega-$ параметр повреждённости, $\varepsilon_{c}-$ эквивалентная деформация, при которой происходит разрушение. Перейдём от интегральной (6) к дифференциальной форме:

$$
\frac{d \omega}{d \varepsilon}=1-\beta
$$

Для построения эволюционного уравнения для $\omega$ проведём ряд преобразований:

$$
\frac{d \omega}{d \varepsilon}=\frac{d \omega}{d \varepsilon} \frac{d t}{d t}=\frac{d \omega}{d t} \frac{d t}{d \varepsilon}=\frac{d \omega}{d t} \frac{1}{\dot{\varepsilon}_{0}} .
$$

Подставив (8) и (5) в выражение (7) получим уравнение для повреждённости:

$$
\dot{\omega}=\dot{\varepsilon}_{0} \frac{(\partial \Psi / \partial p) \dot{p}}{\sigma\left(\dot{\varepsilon}^{p}+\dot{p}\right)},
$$

а условие разрушения запишется как $\omega \geq \omega_{c}$. Параметр $\omega_{c}$ отражает критическое значение накопленной энергии, при котором происходит разрушение материала. Ранее выдвигалась гипотеза о том, что данная величина является константой [11], однако это не так. Согласно структуре уравнений (1) - (3), (9) значение $\omega_{c}$ неявным образом зависит от скорости деформации.
Тем не менее, критерий разрушения остаётся достаточно универсальным и практически не зависит от условий нагружения, о чём свидетельствуют результаты моделирования. Поэтому константа $\omega_{c}$ может быть определена из эксперимента на одноосное растяжение и применена к моделированию процесса усталостного разрушения.

При моделировании усталостного нагружения в задаче (1)-(3),(9) напряжения задавали в виде гармонического закона, аналогично тому, как происходит нагружение в эксперименте: $\sigma=\sigma_{A} \cdot \sin (2 \pi v t)$, где $t-$ время, $\sigma_{A}$ и $\nu=20$ кГц - амплитуда и частота нагружения соответственно.

Идентификацию неизвестных параметров модели проводили в несколько этапов. На первом этапе решали задачу минимизации невязки между расчётной и экспериментальной диаграммами деформирования при квазистатическом нагружении. При этом определяли параметры $\Gamma_{\sigma}, \Gamma_{p \sigma}$ и $\Gamma_{p}$. Затем с использованием полученных параметров минимизировали разницу между расчётной и экспериментальной диаграммами деформирования при динамическом нагружении. При этом определяли параметры $n_{\varepsilon}$ и $n_{p}$. Параметр $\omega_{c}$, как указано выше, может быть определён из эксперимента на одноосное растяжение до момента разрушения с регистрацией тепловыделений и дальнейшим вычислением запасённой энергии. При идентификации использовали как оригинальные эксперименты, так и литературные данные [12]. Идентифицированные параметры модели для сплава АМг6 имеют следующие значения: $\Gamma_{\sigma}=529 \quad(\Pi a \cdot c)^{-1}, \quad \Gamma_{p \sigma}=38.5 \quad(\Pi a \cdot c)^{-1}, \quad \Gamma_{p}=2.9 \quad(\Pi a \cdot c)^{-1}$, $n_{\varepsilon}=n_{p}=0.967, \omega_{c}=0.0432$.

Рассмотрена задача усталостного разрушения сплава АМг6 в исходном состоянии, а также после предварительного динамического нагружения. Сравнение расчётов проводили с экспериментальными данными, полученными в настоящей работе, а также данными других авторов [13]. Для моделирования усталостного разрушения в уравнении (9) в качестве начального условия задавали значение $\omega(0)=0$ при отсутствии преднагружения и $\omega(0)=\int((\partial \Psi / \partial p) \dot{p}) / \sigma\left(\dot{\varepsilon}^{p}+\dot{p}\right) d \varepsilon-$ при наличии предварительной деформации, равной $\varepsilon$. При этом для расчёта предварительного деформирования решали отдельную задачу с соответствующими условиями нагружения. Результаты расчётов и экспериментов представлены на Рис. 4.

По Рис. 4 видно, что предложенная модель хорошо предсказывает усталостную долговечность сплава АМг6, в том числе - после предварительного динамического нагружения.

\section{5. Выводы}

Проведённые исследования показывают, что одной из причин формирования подповерхностных трещин в сплаве АМг6 может служить наличие интерметаллидных включений типа $\mathrm{Al}_{52} \mathrm{Mg}_{48}$. Однако, очаг разрушения в объёме материала может формироваться и без включения - на некотором внутреннем дефекте. Построенные определяющие соотношения в совокуп- 


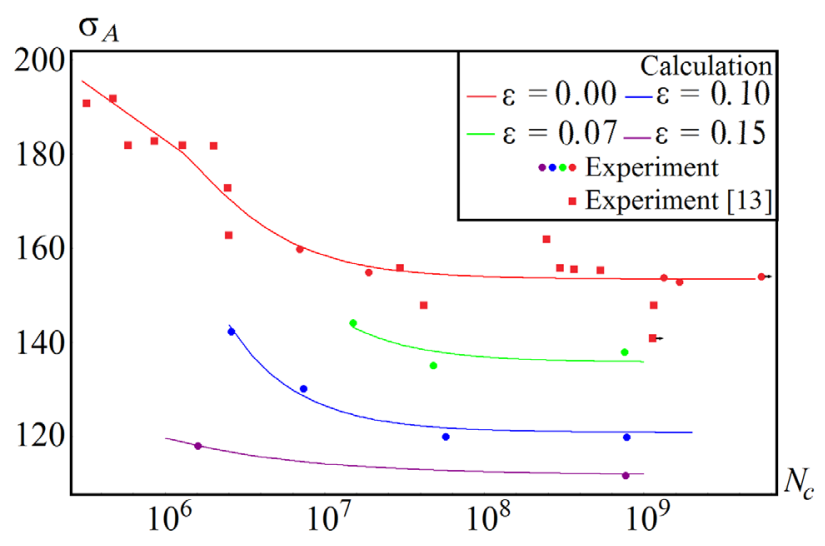

Pис. 4. (Color online) S-N-диаграммы: расчёт и эксперимент. Fig. 4. (Color online) S-N diagrams: calculation and experiment.

ности с предложенным критерием накопления повреждённости способны дать адекватную оценку предела выносливости сплава АМг6 на базе $10^{6}-10^{9}$ циклов. Модель также способна прогнозировать усталостное разрушения после динамических воздействий.

Благодарности /Acknowledgements. Работа выполнена при поддержке гранта Российского научного фонда № 19-79-00168. / This work was supported by the Russian Science Foundation, grant No. 19-79-00168.

\section{Литература/References}

1. H. Mughrabi. Int. J. Fatigue. 28, 1501 (2006). $\underline{\text { Crossref }}$

2. I. N. Fridlyander. Metal Science and Heat Treatment. 44 (7), 292 (2002). Crossref

3. N.S. Zhmuydin, E. A. Bem, D. E. Grishkov, S. V. Nazarov. Actual problems of aviation and astronautics. 1 (14), 432 (2018). (in Russian) [Н. С. Жмуйдин, Е.А. Бем,
Д.Е. Гришков, С. В. Назаров. Актуальные проблемы авиации и космонавтики. 1 (14), 432 (2018).]

4. V. Oborin, M. Sokovikov, D. Bilalov, O. Naimark. Procedia Structural Integrity. 2, 1063 (2016). $\underline{\text { Crossref }}$

5. N.P. Lyakishev. Diagrammy sostoyaniya dvoynykh metallicheskikh sistem. V. 1. Moscow, Mashinostroenie (1996) 992 p. (in Russian) [Н. П. Лякишев. Диаграммы состояния двойных металлических систем. Т. 1. Москва, Машиностроение (1996) 992 с.]

6. A. A. Shanyavskiy, A.P. Soldatenkov. PNRPU Mechanics Bulletin. 1, 198 (2019). (in Russian) [А. А. Шанявский, А. П. Солдатенков. Вестник ПНИПУ. Механика. 1, 198 (2019).] Crossref

7. A. A. Shanyavskiy. Engineering Fracture Mechanics. 110, 350 (2013). Crossref

8. O. B. Naimark, M. V. Bannikov. Letters on materials. 5 (4), 497 (2015). (in Russian) [О. Б. Наймарк, М.В.Банников. Письма о материалах. 5 (4), 497 (2015).] Crossref

9. D.A. Bilalov, M.A. Sokovikov, Yu.V. Bayandin, V.V. Chudinov, V.A. Oborin, O.B. Naimark. PNRPU Mechanics Bulletin. 2, 5 (2019). (in Russian) [Д.А. Билалов, М.А. Соковиков, Ю.В. Баяндин, В. В. Чудинов, В.А. Оборин, О.Б. Наймарк. Вестник ПНИПУ. Механика. 2, 5 (2019).] ㄷossref

10. O. B. Naimark, Y.V. Bayandin, M.A. Zocher. Physical Mesomechanics. 20 (1), 10 (2017). Crossref

11. A. Kostina, O. Plekhov. Theoretical and Applied Fracture Mechanics. 93, 56 (2018). $\underline{\text { Crossref }}$

12. B. L. Glushak, O. N. Ignatova, V. A. Pushkov, S. A. Novikov, A.S. Girin, V.A. Sinitsyn. Journal of Applied Mechanics and Technical Physics. 41 (6), 1083 (2000). Crossref

13. T.Yu. Yakovleva, L.E. Matokhnyuk. Problems of strength. 4, 145 (2004). (in Russian) [Т. Ю. Яковлева, Л.Е. Матохнюк. Проблемы прочности. 4, 145 (2004).] 